\title{
A KINETIC DESCRIPTION OF ELECTRON BEAM EJECTION FROM SPACECRAFT
}

\author{
George V. Khazanov and Torsten Neubert \\ Space Physics Research Laboratory, Department of Atmospheric, Oceanic and \\ Space Sciences, University of Michigan
}

Grigorii D. Gefan and Aleksander A. Trukhan

Irkutsk Polytechnical Institute, Irkutsk, Russia

Evgeni V. Mishin

IZMIRAN, Troitsk, Moscow Region, Russia

\begin{abstract}
A model based on the Boltzmann kinetic equation describing electron beam ejection from spacecraft is presented. Results are shown for steady-state, beam-atmosphere interaction (BAI) and for beam-plasma interaction (BPI). The BPI considered is that of elastic scattering of beam electrons with strong, short-scale Langmuir turbulence. This mechanism has been suggested for prompt echoes of beam electrons observed in sounding rocket experiments. It is shown that the interaction is sufficiently strong to account for observations of back-scattered electrons at the beam accelerator energy. However, it is clear from observations that particles undergo diffusion in energy at a rate exeeding levels expected for BAI. Inelastic scattering in BPI must therefore also be important.
\end{abstract}

\section{Introduction}

Active space experiments involving the ejection of energetic electron beams in the ionosphere have been performed for more than two decades. In beam experiments a variety of phenomena are explored, including auroral emissions [Burch et al., 1993; Mende et al., 1993] and parallel electric fields on auroral fieldlines [Kellogg, 1992]. It is important in such experiments to have an accurate description of the beam dynamics as it propagates away from the spacecraft, that is, the energy degradation and scattering due plasma instabilities, spacecraft charging, etc. While many aspects of beam ejection are well understood, the beam-plasma interaction (BPI) is lacking an accurate description [Neubert and Banks, 1992]. In BPI the bearn drives plasma wave modes and subsequent scattering of beam electrons and heating of the ambient plasma. The high-resolution measurements of wave and particle spectra taken in the last decade from the space shuttle /Gurnett et al., 1986; Oberhardt et al., 1993 and from multi-platform sounding rocket payloads [Winckler et al., 1989; J. Raitt, unpublished manuscript,1993] allow for the creation of better models. These are also needed to better understand specific observations such as the so-called prompt electron echo (PEE) events in rocket experiments [Hendrikson et al., 1975; Winckler et al., 1975; Maehlum et al., 1980; Wilhelm et al., 1985] where echoes are observed with time delays $<100 \mathrm{~ms}$ during upward beam injection, and dischargelike events observed from the space shuttle [Sasaki et al., 1985; Waterman et al., 1988]. To describe BPI quantitatively, a full kinetic treatment of the electron beam and the background plasma is needed in order to determine growth rates of plasma waves and to estimate the feed-back from waves to particles through scattering and diffusion.

Here we present a first step in this direction, namely a kinetic model of electron beam ejection based on the Boltzmann equation. The model is applied to the case of collisional interaction

\section{Copyright 1993 by the American Geophysical Union.}

Paper number 93GL01981

0094-8534/93/93GL-01981\$03.00 with the ambient neutral atmosphere, the beam-atmosphere interaction (BAI) [Neubert et al., 1990] and to the case of elastic scattering of beam electrons by short-scale Langmuir turbulence in the beam. This choice was motivated by the observations of PEE events mentioned above. We use the formulas of Mishin [1983] and Mishin et al. [1989], who suggested that PEE can be interpreted in terms of the theory of BPI developed for active experiments [Galeev et al., 1976; Mishin and Ruzhin, $1980,1981]$. This theory considers the regime of Strong Turbulence (ST), which is characteristic for BPI at high altitudes where the major part of the energy of Langmuir waves concentrates in the short-scale spectral region. The phase velocities of the short-scale waves are much less than that of the beam. Therefore the wave-particle interaction preserves, in the first approximation, the particle energy, i.e., the particle is scattered elastically.

\section{The Kinetic Beam Model}

Suprathermal electrons of the ionosphere and plasmasphere are represented by the distribution function $f(t, \vec{r}, \vec{v})$ which satisfies the kinetic equation

$$
\frac{\partial f}{\partial t}+\vec{v} \vec{\nabla} f+\frac{\vec{F}}{m} \vec{\nabla}_{\vec{v}} f=S_{e e}+S_{e i}+S_{e n}+S_{e n}^{*}+S_{e n}^{+}+S_{e p}+q
$$

where $F=-e(\vec{E}+\vec{v} \times \vec{B} / c)$ is the Lorentz force on an electron from the electric and magnetic fields $\vec{E}, \vec{B}$, and $S$ represents the collision terms: elastic collisions with thermal electrons, $S_{e e}$, ions $S_{e i}$ and neutral particles $S_{e n}$, and inelastic collisions giving rise to the excitation of structural levels of atoms and molecules $S_{e n}^{*}$ and ionization $S_{e n}^{+}$. The source of electrons from ionization or from a beam source is $q$, and $S_{e p}$ is the electronplasmon collision term describing the BPI.

Introducing in velocity space a spherical coordinate system with the polar axis along the magnetic field it is possible to obtain the kinetic equation in the drift approximation [Khazanov, 1979]

$$
\begin{gathered}
\frac{\partial\langle f\rangle}{\partial t}+\mu v \frac{\partial\langle f\rangle}{\partial s}-\frac{1-\mu^{2}}{v}\left(\frac{e}{m} E_{\|}+\frac{v^{2}}{2 B} \frac{\partial B}{\partial s}\right) \frac{\partial\langle f\rangle}{\partial \mu} \\
-\frac{e}{m} E_{\|} \mu \frac{\partial\langle f\rangle}{\partial v}=\langle S\rangle
\end{gathered}
$$

where $\mu=\cos \theta, s$ is the coordinate along the geomagnetic field, $E_{\|}$is the longitudinal component of the electric field, and $\langle S\rangle$ is a symbol representing the right-hand side of (1). Brackets denote the average over the azimuth angle as shown for $f$ :

$$
\langle f\rangle=\frac{1}{2 \pi} \int_{0}^{2 \pi} f(\phi) d \phi
$$


In the case of interest one may drop the small terms associated with the plasma transport across the magnetic field as well as making no distinction between the distribution function and the guiding center distribution function. Therefore, in what follows we shall omit the brackets around the distribution function.

It is convenient to pass from the distribution function to the flux $\psi=2 E f / m^{2}$ defined such that $\psi(t, \vec{r}, E, \theta, \phi) d E d \Omega$ is the number of electrons with the energy from $E$ to $E+d E$ with the direction of velocity $\vec{v}$ inside the solid angle $d \Omega=\sin \theta d \theta d \phi$ that meet at the point $(t, \vec{r})$ per unit time per unit area.

The collision terms in (2) may be brought to the form

$$
\begin{gathered}
\left\langle S_{e e}\right\rangle+\left\langle S_{e \imath}\right\rangle=\frac{A v n_{e}}{2 E^{2}} \frac{\partial}{\partial \mu}\left[\left(1-\mu^{2}\right) \frac{\partial f}{\partial \mu}\right]+\frac{A v n_{e}}{E} \frac{\partial f}{\partial E} \\
S_{e e}=\frac{v n_{n} \sigma_{n}^{t}}{2} \frac{\partial}{\partial \mu}\left[\left(1-\mu^{2}\right) \frac{\partial f}{\partial \mu}\right] \\
\langle S\rangle_{e n}^{*}=\frac{n_{n} v}{E} \sum_{\jmath} E_{n j} \frac{\partial}{\partial E}\left[E \sigma_{n j}^{*}(E) f(E, \mu, s)\right] \\
\langle S\rangle_{e n}^{+}=\frac{n_{n} v}{E} \frac{\partial}{\partial E}\left[\langle E\rangle \sigma_{n}^{+}(E) f(E, \mu, s)\left(E_{n}^{+}+\hat{E}_{n}\right)\right] \\
+\frac{n_{n} v}{E} \int_{2 E+E_{n}^{+}}^{\infty} E I_{n}^{+}\left(E^{\prime}, E\right) f_{o}\left(E^{\prime}, s\right) d E^{\prime}
\end{gathered}
$$

Here $n_{e}$ is the density of electrons and and $n_{n}$ the densities of neutral particles of species $n, \sigma_{n}^{t}$ is the transport cross-section of elastic scattering, $\sigma_{n j}^{*}$ is the inelastic cross-section of scattering that characterizes the transition of a neutral particle to an excited state with energy $E_{n j}^{*}, E_{n}^{+}$is the energy of ionization, $A=2 \pi e^{4} C_{l}$ ( $C_{l}$ being the Coulomb logarithm). $\sigma_{n}^{+}$is defined as

$$
\sigma_{n}^{+}(E)=\int_{0}^{\left(E-E_{n}^{+}\right) / 2} I_{n}^{+}\left(E, E_{2}\right) d E_{2}
$$

and is the full cross-section of ionization by an electron with energy $E$. The quantity $I_{n}^{+}\left(E, E_{2}\right)$ represents the corresponding differential cross section, where $E_{2}$ is the energy of the secondary electron, and

$$
\hat{E}_{n}=\frac{1}{\sigma_{n}^{+}} \int_{0}^{\left(E-E_{n}^{+}\right) / 2} E_{2} I_{n}^{+}\left(E, E_{2}\right) d E_{2}
$$

is the mean energy of a secondary electron. Finally,

$$
f_{o}\left(E^{\prime}, s\right)=\frac{1}{2} \int_{-1}^{1} f\left(E^{\prime}, s, \mu\right) d \mu
$$

The approximations used to derive (4)-(6) are discussed in detail in [Krinberg,1978; Khazanov,1979]. The accuracy of these approximations is quite sufficient for the investigation discussed in this paper.

The final element needed is the collision frequency of beam electrons with the strong wave turbulence. Consider the interaction of a keV-energy electron beam with an isotropic distribution of short-scaled Langmuir waves. The phase velocities of such waves, $\omega / k$, are much less than the beam velocity, i.e. $\delta=\omega / k / v_{o}<<1$, where $v_{o}$ is the velocity of beam electrons.
This situation corresponds to the strong turbulence approach in BPI [Sagdeev, 1979; Shapiro and Shevchenko, 1984], where the major part of the turbulence concentrates in the short-scale spectral region $k \simeq k_{L} \gg k_{o}=\omega_{p} / v_{o}$ ( $\omega_{p}$ is the electron plasma frequency). Note, that isotropy of the turbulence is provided by chaotic orientation in space of the plasma cavities where the Langmuir waves are trapped. Also, it is essential that the scalelength of isotropization of the beam by short-scale interactions is assumed to be much larger than the relaxation length of the beam due to BPI with the large-scale, $k \simeq k_{o}$, waves [Sagdeev, 1979|. This allows for determining the parameters characteristic for the "elastic" scattering through the parameters of BPI. Thus, one gets the electron-plasmon collisional term [Mishin et al., 1989, 1990]

$$
\langle S\rangle_{e p}=\nu_{e f f} \frac{\partial}{\partial \mu}\left[\left(1-\mu^{2}\right) \frac{\partial f}{\partial \mu}\right]
$$

The effective frequency $\nu_{\text {eff }}$ in (7) of electron-plasmon interaction is found from

$$
\nu_{e f f}=\omega_{p}\left(\frac{W_{L}}{n_{e} T_{e} k_{\perp} r_{D}}\right)\left(\frac{T_{e}}{E_{b}}\right)^{3 / 2}
$$

Where $W_{L}$ is the energy density of plasma waves in the region of turbulence, and the main part of the wave energy is concentrated near $k_{L}=\sqrt{W_{L} / n_{e} T_{e} r_{D}^{2}}$. Expressing $W_{L}$ from the energy balance equation in the beam-plasma system, one can get [Mishin et al., 1989,1990]

$$
\nu_{e f f} \simeq 1.1 \omega_{p}\left(\frac{n_{b}}{n_{e}}\right)^{1 / 2}\left(\frac{u}{\Delta u}\right)\left(\frac{T_{e}}{E_{b}}\right)^{3 / 2}
$$

At values of $n_{b} / n_{e}=10^{-4}-10^{-1}, u / \Delta u \simeq 10, T_{e} / E_{b} \simeq 10^{-2}$, $\omega_{p} \simeq 10^{7} \mathrm{rad} / \mathrm{sec}^{-1}, E_{b}=\left(m_{e} u^{2}\right) / 2 \simeq 3-10 \mathrm{keV}$, we get $\nu_{e f f} \simeq$ $10^{3}-10^{5} \mathrm{sec}^{-1}$, the dependence of $\nu_{\text {eff }}$ on the altitude being weak.

\section{Results}

Model calculations have been performed with the electron beam source located at $160 \mathrm{~km}$ altitude. The magnetic field is assumed to be directed upwards, corresponding to an experiment loaction in the Southern hemisphere, with beam electrons ejected within a cone of pitch-angles around the magnetic field of $(0, \pi / 15)$ and $(15 \pi / 16, \pi)$ for upward and downward injection respectively. The energy spectrum of beam electrons was given on the form

$$
\psi(E)=10^{2} E \exp \left(-\left|E-E_{b}\right| / E_{o}\right)
$$

with $E_{b}=10 \mathrm{keV}$ and $E_{o}=1 \mathrm{keV}$.

The value of the numerical coefficient can be set arbitrarily, because by virtue of the linearity of the kinetic equation (2) the solution can easily be recalculated for any other source intensity.

The problem is solved along a closed magnetic field-line from $100 \mathrm{~km}$ altitude in one hemisphere to $100 \mathrm{~km}$ altitude in the conjugate hemisphere. Figure 1 shows the ratio of the downward $\Phi^{-}$and upward $\Phi^{+}$electron flux as a function of energy at the spacecraft altitude for upward beam ejection, where $\Phi^{+}, \Phi^{-}$ are the hemispherical fluxes defined as

$$
\Phi^{-}(E)=\int_{-1}^{0} \mu \psi(E, \mu) d_{\mu}
$$




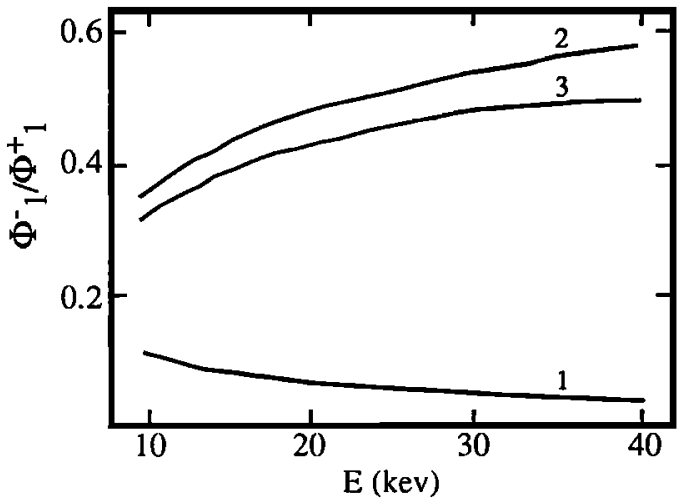

Fig. 1. Albedo at the source altitude $(160 \mathrm{~km})$ for upward injection: 1. $\nu_{e f f}=0 \mathrm{~s}^{-1} ; 2 . \nu_{e f f}=10^{3} \mathrm{~s}^{-1} ; 3 . \nu_{e f f}=10^{4}$ $\mathrm{s}^{-1}$.

$$
\Phi^{+}(E)=\int_{0}^{1} \mu \psi(E, \mu) d \mu
$$

The relationship $\Phi^{-} / \Phi^{+}$has the meaning of albedo and characterizes the reflective ability of the region above the injector. In the absence of BPI scattering, $\left(\nu_{e f f}=0\right)$ (curve 1), the albedo is small and decreases with increasing energy of ejected electrons. This behaviour reflects the decrease with energy of the electron-neutral elastic cross-section. When scattering with plasmons is included, however, the albedo increases sharply. In this case, two features are noted. Firstly, the albedo increases with electron energy, and secondly, as the effective scattering frequency increases from $10^{3} \mathrm{~s}^{-1}$ (curve 2) to $10^{4} \mathrm{~s}^{-1}$ (curve 3) the albedo does not increase as might be expected, but decreases. Both these effects are related to the fact that during strong pitch-angle scattering, when the transverse velocity of the injected particles grows at the expense of the longitudinal one, the electron trajectory changes substantially and energy loss during inelastic collisions with neutrals become increasingly important. This leads to the decrease of albedo at too high values of $\nu_{\text {eff }}$. The cross-section for electron-neutral inelastic collisions decreases with increasing electron energy. As a result, inelastic collisions become less important at higher energies and the albedo increases with energy.

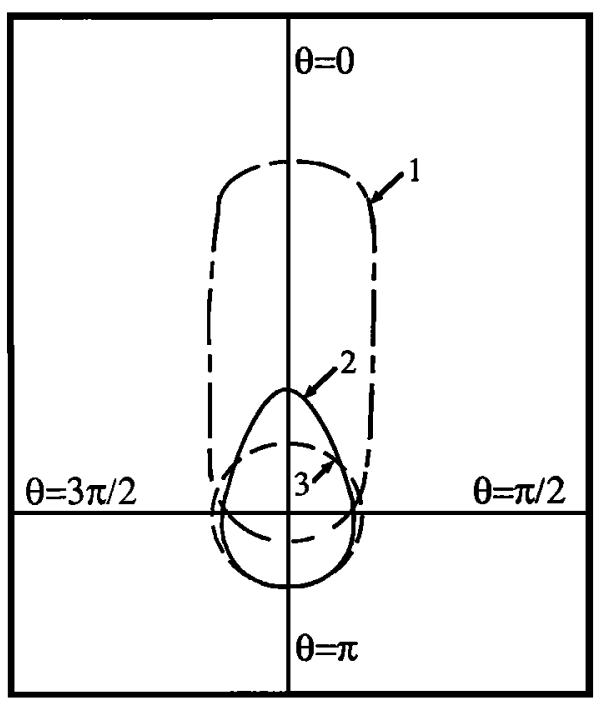

Fig. 2. Pitch-angle distribution $20 \mathrm{~km}$ above the source altitude for upward injection: $1 . \nu_{\text {eff }}=0 \mathrm{~s}^{-1} ; 2 . \nu_{\text {eff }}=10^{3} \mathrm{~s}^{-1} ; 3$. $\nu_{\text {eff }}=10^{4} \mathrm{~s}^{-1}$.
The pitch angle distribution $20 \mathrm{~km}$ above the source altitude is illustrated in Figure 2 for upward ejection. Curve 1 is an isocontour for the case of no BPI, showing a distribution function extended in the direction of the ejected beam velocity component. It reflects the steady-state distribution function of the BAI. The beam character of the source is clearly seen, though modified by the collisional interactions with the ambient neutral atmosphere. When the scattering with plasmons is included, the distribution function becomes increasingly isotropized and the beam character is lost.

The ion production rate as a function of altitude is shown in Figure 3. Curves 1-3 are for upward injection for $\nu_{\text {eff }}=$ $0,10^{3} \mathrm{~s}^{-1}, 10^{4} \mathrm{~s}^{-1}$ respectively. As BPI is included, the production rate increases by a factor of $2-3$. This is caused by the increase in path-length experience by energetic electrons as they are elastically scattered by the wave turbulence. For reference, downward injection with no BPI is shown with curve 4 .

\section{Discussion}

The expression for $\nu_{e f f}$ in (7) contains both beam and ambient plasma parameters. The greatest uncertainty in the estimate of $\nu_{\text {eff }}$ is the value of $T_{e}$, which is higher than the ionospheric electron temperature because of heating by plasma waves generated as a result of the beam ejection. According to experimental data [Gringauz et al., 1981], the background electron temperature during the injection of powerful electron beams can reach the value of about $100 \mathrm{eV}$, which was used for obtaining an estimate of $\nu_{e f f}$.

The numerical solutions to the kinetic equation confirm that short-scale Langmuir turbulence can generate substantial scattering of injected beams as suggested by Mishin [1983, 1990]. It is clear from experiments performed in the last decade, however, that the back-scattered electron flux is a result of processes that involve diffusion in energy as well as in pitch-angle and therfore cannot be described by an elastic scattering alone. Typical electron spectra have inverse power-law dependence with energy, with energies reaching from the eV-range to above the accelerator energy. It is clear, therefore, that the elastic scattering considered here cannot be the sole source of backscattered electrons. Our future work will include a more complete description of the BPI.

As mentioned earlier, the albedo shown in Figure 1 increases with decreasing energy for the case of BAI alone because the elastic cross-section with neutrals also increases with decreasing energy. When BPI is included, this trend is reversed and the albedo decreases with decreasing energy. It is expected that at beam energies below a few $\mathrm{keV}$, for the conditions modeled here, that the BAI will dominate over the BPI. This is consistent with

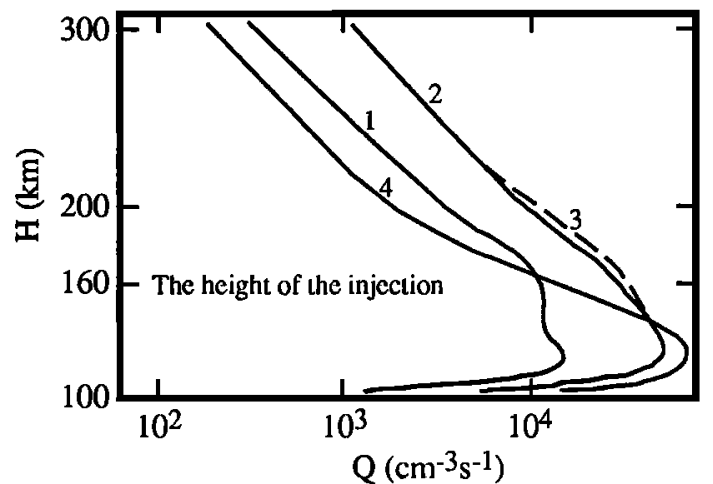

Fig. 3. The ion production rate as a function of altitude. Upward injection: 1. $\nu_{\text {eff }}=0 \mathrm{~s}^{-1} ; 2 . \nu_{e f f}=10^{3} \mathrm{~s}^{-1} ; 3$. $\nu_{e f f}=10^{4} \mathrm{~s}^{-1}$. Downward injection: 4. $\nu_{e f f}=0 \mathrm{~s}^{-1}$. 
conclusions reached from sounding rocket observations of return current measurements during beam ejection [Neubert et al., 1990]. Here it was found that BAI-induced fluxes, together with "passive" currents from the ambient ionosphere, could account for the observed currents.

Acknowledgments. This work was supported by NASA contracts NAS8-38772 and NAS8-39381, NASA grants NAGW1619 and NAG5-1500, NSF grant ATM-9114409, and AFOSR grant F19628-93-k-003.

\section{References}

Burch, J.L., S.B. Mende, N. Kawashima, W.T. Roberts, W. W. L. Taylor, T. Neubert, W.C. Gibson, J.A. Marshall, and G.R. Swenson, Artificial auroras in the upper atmosphere, 1, Electron beam injections, Geophys. Res. Lett., 20, 491, 1993.

Galeev, A.A., E.V. Mishin, R.Z. Sagdeev, V.D. Shapiro, and V.I. Shevchenko, Discharge in the region around a rocket following the injection of electron beams into the ionosphere, Sov. Phys. Doklady, 231, 71, 1976.

Gringaus, K.I., E.V. Mishin, and N.M. Shutte, Rocket potential measurements during electron beam injection into the ionosphere, Adv. Space Res., 2, 69, 1981.

Gurnett, D.A., W.S. Kurth, J.T. Steinberg, P.M. Banks, R.I. Bush, and W.J. Raitt, Whistler-mode radiation from the Spacelab-2 electron beam, Geophys. Res. Lett., 13, 225, 1986.

Hendrikson, R.A., R.W. McEntire, and J.R. Winckler, ECHO1: an experimental analysis of local effects and conjugate return echoes from an electron beam injection into the magnetosphere by a sounding rocket, Planet. Space Sci., 23, 1431, 1975.

Oberhardt, M.R., D.A. Hardy, I. Katz, M.P. Gough, and D.C. Thompson, Vehicle charging as measured by the shuttle potential and return electron experiment on TSS-1, AIAA 930699, January, 1993.

Kellogg, P.J., Electron beams and their interactions with the ionosphere- a review of the E \| B series, Adv. Space Res., 12, No.12, (12)15, 1992.

Khazanov, G. V., Kinetics of plasma electron component in the upper atmosphere, Moscow, Nauka, 1979.

Krinberg, I. A., Kinetics of electrons in the Earth's ionosphere and plasmasphere, Moscow, Nauka, 1978.

Maehlum, B.N., B. Grandal, T.A. Jacobsen, and J. Troim, Polar 5- an electron accelerator experiment within an aurora: 2. Scattering of an artificially produced electron beam in the atmosphere, Planet. Space Sci., 28, 279, 1980.

Mende, S.B., J.L. Burch, G.R. Swenson, E.K Aamodt, S.P. Geller, R.L. Rairden, and P.L. Hassler, Artificial auroras in the upper atmosphere: 2, Imaging results, Geophys. Res. Lett., 20, 495, 1993.

Mishin, E.V, On macroscopic consequences of plasma turbulence excited by charged particle fluxes injected from spacecraft, in: Proc. of Symposium on Physics of the Earth's Ionosphere and Magnetosphere and the Solar Wind, Moscow, IKI,1983.
Mishin, E.V., and Yu.Ya. Ruzhin, The model of beam-plasma discharge in the rocket environment during an electron beam injection in the ionosphere, Ann. Geophys., 36, 432, 1980.

Mishin, E.V., and Yu.Ya. Ruzhin, Beam-plasma discharge in near-rocket region, Adv. Space Res., 1, 47, 1981.

Mishin, E.V., Yu.Ya. Ruzhin, and V.A. Telegin, The Interaction of Electron Beams With Ionospheric Plasma, Leningrad: Gidrometeoizdat, 1989.

Mishin, E.V., A.A. Trukhan, and G.V. Khazanov, Plasma Effects of Superthermal Electrons in the Ionosphere,Moscow, Nauka, 1990.

Neubert, T., and P.M. Banks, Recent results from studies of electron beam phenomena in space plasmas, Planet. and Space Sci., 40, 153, 1992.

Neubert, T., P.M. Banks, B.E. Gilchrist, A.C. Fraser-Smith, P.R. Williamson, W.J. Raitt, N.B. Myers, and S. Sasaki, The interaction of an artificial electron beam with the earth's upper atmosphere: effects on spacecaft charging and the nearplasma environment, J. Geophys. Res., 25, 12209, 1990.

Sagdeev, R.Z., The 1976 Oppenheimer Lectures: Critical problems in plasma astrophysics, Rev. of Mod. Phys., 51, No. 1, 1-20, 1979.

Sasaki, S., N. Kawashima, K. Kuriki, M. Yanagisawa, T. Obayashi, W.T. Roberts, D.L. Reasoner, W. W. L. Taylor, P. R. Williamson, P.M.Banks, and J.L.Burch, Ignition of beam plasma discharge in the electron beam experiment in space, Geophys. Res. Lett., 12, 647, 1985.

Shapiro, V. D., and V. I. Shevchenko, Strong Turbulence of Plasma Oscillations, Handbook of Plasma Physics, Vol. 2, Chapter 5.4, edited by A.A. Galeev and R. N. Sudan, Elsvier Science Publishers B. V., 1984.

Watermann, J., K. Wilhelm, K.M. Torkar, and W. Riedler, Space shuttle charging or beam-plasma discharge: What can electron spectrometer observations contribute to solving the question ?, J. Geophys. Res., 93, 4134, 1988.

Wilhelm, K., W. Bernstein, Paul J. Kellogg, and B.A. Whalen, Fast magnetospheric echoes of energetic electron beams, J. Geophys. Res., 90, 491, 1985.

Winckler, J.R., R.L. Arnoldy and R.A. Hendrikson, Echo 2: A study of electron beams injected into the high- altitude ionosphere from a large sounding rocket, J. Geophys. Res., $80,2083,1975$.

Grigorii D. Gefan and Aleksander A. Trukhan, Irkutsk Polytechnical Institute, 664074 Irkutsk, Russia

George V. Khazanov and Torsten Neubert Space Physics Research Laboratory, Department of Atmospheric, Oceanic, and Space Sciences, University of Michigan, Ann Arbor, MI 481092143.

Evgeni V. Mishin, IZMIRAN, 142092 Troitsk, Moscow Region, Russia.
(Received: May 31, 1993; accepted: July 2, 1993) 